\title{
Pelvic Floor Myofascial Pain Might Influence Treatment Outcome of Interstitial Cystitis/bladder Pain Syndrome
}

\section{Wan-Ru Yu}

Hualien Tzu Chi Hospital, Buddhist Tzu Chi Medical Foundation

\section{Fei-Chi Chuang}

Kaohsiung Chang Gung Memorial Hospital, Chang Gung University College of Medicine

\section{Wei-Chuan Chang}

Hualien Tzu Chi Hospital, Buddhist Tzu Chi Medical Foundation

Hann-Chorng Kuo ( $\nabla$ hck@tzuchi.com.tw)

Hualien Tzu Chi Hospital, Buddhist Tzu Chi Medical Foundation

\section{Research Article}

Keywords: interstitial cystitis/bladder pain syndrome; pelvic floor muscle, myofascial pain, trigger point, vaginal digital examination, treatment outcome

Posted Date: December 29th, 2021

DOI: https://doi.org/10.21203/rs.3.rs-1176386/v1

License: (c) (i) This work is licensed under a Creative Commons Attribution 4.0 International License. Read Full License 


\section{Abstract}

Introduction

In patients with interstitial cystitis or bladder pain syndrome (IC/BPS), $85 \%$ were found to have pelvic floor myofascial pain (PFMP) and hypertonicity (PFH). However, they are not typically trained to consider or assess PFMP as a contributing factor to patients' IC/BPS symptoms. This study aimed to explore the relationship between PFMP and treatment outcomes in women with IC/BPS.

Methods

Patients with IC/BPS who received any type of treatment were prospectively enrolled. They underwent vaginal digital examination at baseline. PFMP severity was quantified on the visual analog scale (VAS). Subject assessment items included O'Leary-Sant symptom score (OSS), Global Response Assessment (GRA), and Beck's anxiety inventory. Object assessment items included bladder computed tomography (CT), urodynamic parameters, maximum bladder capacity, and grade of glomerulation.

Results

A total of 65 women with IC/BPS (mean age, $57.1 \pm 11.3$ years) were enrolled in the study. Patients with more severe PFMP had significantly higher rate of dyspareunia $(p=.031)$; more comorbidities $(p=.010)$; higher number of PFMP sites $(p<.001)$; and higher OSS $(p=.012)$. PFMP severity was not significantly correlated with bladder conditions, whether subjective or objective. Moreover, PFMP severity (VAS) was significantly negatively associated with GRA score.

Conclusion

PFMP might affect the subjective results of IC/BPS treatment but not the bladder condition. Therefore, in the future treatment of patients with IC/BPS, digital vaginal examinations of pelvic floor muscles should be performed and focused more on the PFM-related conditions, and necessary PFM treatments, such as the vaginal pelvic floor muscle message, should be scheduled.

\section{Introduction}

Interstitial cystitis/bladder pain syndrome (IC/BPS) is a disease of unknown etiology. This multifactorial clinical condition is typically characterized by irritative voiding symptoms in the form of urinary frequency and urgency, with debilitating episodic chronic pain. ${ }^{1}$ IC/BPS has been included in myofascial pain and neuropathic pain syndromes interrelated with the immune and inflammatory systems. ${ }^{2}$ The prevalence rate of IC/BPS is between $3 \%$ and $10 \%$ and more frequent among women. ${ }^{3}$ To date, classic Hunner's lesion interstitial cystitis (HIC) is a bladder disease, and bladder treatment improves symptoms. NonHunner's lesion interstitial cystitis (NHIC) likely has multiple etiologies and, in many cases, is difficult to treat. The bladder might be an innocent bystander in a larger pelvic process, ${ }^{4}$ and systemic and immune 
conditions are in need of further attention. Jiang et al. even pointed out that suburothelial inflammation and apoptosis are highly prevalent in the bladders of patients with urolithiasis, which even had a smaller maximal bladder capacity (MBC). ${ }^{5}$ Our previous study also demonstrated that stress is positively associated with pain in patients with IC/BPS, while stress reduction can lead to a simultaneous decrease in the severity of other symptoms. ${ }^{6}$

Pelvic floor muscle (PFM) is often associated with urological, gynecological, gastrointestinal, and sexual problems and chronic pelvic pain. ${ }^{8}$ Several researchers also noted that PFM hypertonicity (PFH), also called pelvic floor myofascial pain (PFMP), could affect treatment outcome and quality of life. ${ }^{2,7-9}$ The prevalence of PFMP in patients with IC/BPS ranges from $50 \%$ to $90 \% .{ }^{4}$ When muscle fiber trauma occurs, inflammatory mediators, such as bradykinin, serotonin, prostaglandins, adenosine triphosphate, and histamine, are released locally, which sensitize muscle nociceptors and reduce their mechanical threshold and result in muscle hyperalgesia and mechanical allodynia. ${ }^{10}$ Myofascial pain can also develop secondary to the presence of bladder pain syndrome. ${ }^{11}$ The location and severity of PFMP are significantly correlated with degree of symptoms, even after controlling for postmenopausal status. ${ }^{9}$

The PFMP may be quite severe and is usually accompanied by acute attacks that awake patients, and symptoms are usually vague and poorly localized. ${ }^{12}$ However, pelvic pain is not perceived at the site of the trigger point origin but at a remote site (referred pain). ${ }^{11,13}$ Until very recently, these hypotheses were difficult to study because a standardized, reproducible examination for PFMP did not exist. ${ }^{6} \mathrm{In}$ patients with IC/BPS, $85 \%$ were found to have PFMP and PFH. ${ }^{14}$ However, PFMP has remained largely understudied and poorly recognized by women's health providers who are not typically trained to consider or assess for PFMP as a contributing factor to patients' IC/BPS symptoms. ${ }^{6}$ This study aimed to explore the relationship between PFMP and treatment outcomes in women with IC/BPS.

\section{Methods}

This is a prospective study that enrolled 65 women who had been diagnosed with IC/BPS from September 2020 to June 2021. The Ethics Committee of Buddhist Tzu Chi General Hospital had approved the study. (IRB Number: IRB105-25-B). All methods were performed in accordance with the relevant guidelines and regulations. Patients were informed about the study rationale and procedures; written informed consent was obtained from all patients prior to enrolment and treatment. These women had been evaluated and treated by urologists, gynecologists, psychiatrist, and other medical specialists without resolution of their pain. The diagnosis of IC/PBS was established based on the characteristic symptoms and cystoscope findings of glomerulations, petechiae, or mucosal fissures after hydrodistention. ${ }^{15}$ They had received several treatments, including cystoscopic hydrodistention, intravesical botulinum toxin A injection, electrocoagulation of Hunner's lesions, and platelet-rich plasma (PRP) intravesical injection. All patients had received video urodynamic study (VUDS),

bladder computed tomography (CT), and cystoscopic hydrodistention at baseline. ${ }^{15}$ Vaginal digital 
examination was performed at the first visit to evaluate their PFM condition before treatment. The patients were investigated thoroughly upon enrolment and excluded if they did not meet the inclusion criteria of the National Institute of Diabetes and Digestive and Kidney Diseases. ${ }^{15}$

Our evaluation included a comprehensive history and pelvic examination performed by a certified nurse practitioner using the right hand with forefinger to examine all women in a comfortable dorsal lithotomy position. ${ }^{16}$ The pelvic examination included assessment of pubovaginalis, puborectalis, iliococcygeus, coccygeus, anal sphincter, obturator internus, piriformis, and tendinous arch. Pain was quantified on a 10point visual analog scale (VAS) by the patient. A retrospective chart review was conducted to gather additional data. Descriptive statistics were used to describe the sample and distribution of variables, such as bladder condition and pain levels.

The evaluation items included patient's bladder characteristics, O'Leary-Sant score (OSS) with IC symptom index (ICSI) and IC problem index (ICPI), bladder CT, urodynamic parameters, MBC, and grade of glomerulation under cystoscopic hydrodistention. Global Response Assessment (GRA) was administered or determined at one month after treatment, and anxiety severity was assessed by the Beck's anxiety inventory (BAI). The BAl score was $0-18$ points, indicating mild anxiety; $19-29$ points indicating moderate anxiety; and 30-63 indicating severe anxiety. ${ }^{17}$

Statistical comparisons between the groups were tested using the Pearson's chi-square test or Fisher's exact test for categorical variables, and an independent t-test or analysis of variance for continuous variables, to use multiple linear regression analyses on factors associated with PFMP and pain severity. All statistical analyses were performed using SPSS (version 22.0, SPSS Inc., Chicago, IL), with a p-value $<.05$ being used as the criterion for statistical significance.

\section{Results}

A total of 65 women with IC/BPS were included in this study. The mean age was $57.1 \pm 11.3$ years, and the mean duration of IC symptoms was $12.8 \pm 10.5$ years. They had received three types of treatment on average, seven (11\%) patients underwent cystoscopic hydrodistention, three (5\%) received intravesical botulinum toxin A injection treatment, 53 (82\%) patients received PRP intravesical injection, and two (3\%) patients received the electrocoagulation of Hunner's lesions treatment. Moreover, 55 (73.8\%) patients have had a vaginal delivery. Regarding sexual behavior, $30(46.1 \%)$ patients had sex within the recent one year, but $31(47.7 \%)$ patients did not have sex for up to five years, and overall, up to $32(49.2 \%)$ patients had pain during sexual intercourse. We also found that 29 (44.6\%) patients had transvaginal surgery. The self-reported mean OSS was $25.9 \pm 7.8$, self-reported lower abdominal pain score under the Numerical Rating Scale (NRS) was $6.1 \pm 2.6$, and mean severity of anxiety using BAI was $21.4 \pm 12.0$. All patients underwent cystoscopic hydrodistention, with a mean MBC of $801.6 \pm 170.6$ $\mathrm{mL}$ and mean glomerulation grade of $1.4 \pm 1.1$. 
When these patients underwent vaginal examination to assess their PFM trigger points, 56 (86.2\%) patients had PFMP. Among the 14 assessment sites, the mean number of painful sites was $5.2 \pm 4.4$, and the mean PFMP severity by VAS score was $6.9 \pm 3.0$ points. One month after treatment, $17(26.1 \%)$ patients reported no change in GRA or even poor change (GRA $=-1 \sim-3$ ), nine (13.8\%) patients had no change after treatment $(\triangle \mathrm{GRA}=0), 20(30 \%)$ patients reported slight improvement $(\Delta \mathrm{GRA}=+1)$, and 28 (43\%) patients reported a significant improvement after treatment $(\triangle G R A=+2 \sim+3)$. The most painful sites are the obturator internus (63.1\%) and puborectalis (60\%) (Figure 1). In the bladder CT of each patient with IC/BPS, we also noted that the bladder wall phenotype of $42(66.7 \%)$ patients is smooth bladder wall. Furthermore, the bladder wall of 21 (33.3\%) patients has a focal thickness or diffuse thickening.

When we divided the vaginal examination PFM trigger point pain score into three groups, 12 patients had mild PFMP (VAS of 0 to 3), 11 patients had moderate PFMP (VAS of 4 to 6), and 42 patients had severe PFMP (VAS of 7-10). Patients with more severe PFMP had significantly higher rate of dyspareunia in the sexual life (16.7\% in the mild pain group, $45.5 \%$ in the moderate pain group, and $59.5 \%$ in the severe pain group, $p=.031$ ); more comorbidities (mild pain group, $7 \pm .7$; moderate pain group, $2.6 \pm 1.7$; severe pain group, $2.2 \pm 1.8$; $p=.010$ ); higher number of myofascial pain sites (mild pain group, $.8 \pm 1.4$; moderate pain group, $3.6 \pm 1.5$; severe pain group, $6.8 \pm 4.4 ; p<.001$ ); and significant correlation with OSS (mild pain group, $19.9 \pm 8.4$; moderate pain group, $27.1 \pm 9.2$; severe pain group, 27.3 \pm 6.6; $p=.012$ ) (Table 1).

Meanwhile, the self-reported treatment outcome (GRA) also had a significant correlation with different PFMP severity groups $(p=.015)$. The GRA score was $2.2 \pm .9$ in patients with mild PFMP, which was higher than that in patients with moderate PFMP $(1.6 \pm .7)$ and severe PFMP $(.9 \pm 1.5)$ (Table 1). However, the PFMP severity was not significantly correlated with the bladder conditions, whether subjective or objective, such as the patient self-reported uncomfortable bladder condition severity (NRS), MBC, and glomerulation grade during cystoscopic hydrodistention, VUDS parameters, pelvic CT image, and even uroflowmetry parameters such as $Q \max (\Delta p$ value $=.176)$, voiding volume $(\Delta p$ value = $.31)$, and post-void residual urine $(P V R)(\Delta p$ value $=.770)$ (Table 2).

The final regression model revealed that the PFMP severity (VAS) as a dependent variable, including six independent variables, of which only GRA $(p<.001)$ was statistically significant, and there was no significant difference with age, OSS, anxiety severity (BAI), glomerulation grade, and MBC. The results showed that an improvement of .10 on the PFMP severity (VAS) of relatives' GRA was associated with a decreased burden of 1.65 scale steps (Table 3 ).

\section{Discussion}

To date, there is no gold standard treatment strategy for patients with IC/BPS, only a suitable treatment. ${ }^{18}$ Many efforts have been attempted to find a better treatment outcome for patients with IC/BPS, including antiviral treatment for Epstein-Barr virus infection, ${ }^{19,20}$ PRP injection for 
increase in regenerative deficits in IC/BPS, ${ }^{19,20}$ and psychiatric consultation for psychosomatic dysfunction associated with IC/BPS. ${ }^{19,20}$ In this study, we also found that patients with IC/BPS have 2.0 \pm 1.7 comorbid psychosomatic disorders, such as depression, insomnia, reflux esophagitis, asthma, irritable colon, and arrhythmia. ${ }^{21}$

Simultaneously, Gisela also reported the high frequency of comorbid disorders well beyond geographic contiguity with the bladder region affecting our pathophysiologic construct of IC/BPS, suggesting either some type of systemic disorder, a process under central nervous system (CNS) control, or both. ${ }^{22} \mathrm{An}$ abnormal CNS drive seems likely to cause a secondary psycho-neuro-endocrine-immune dysfunction in patients with IC/BPS. ${ }^{21}$ Significantly more psychosomatic comorbidities are noted in the moderate PFMP severity group than that in the mild PFMP group $(p=.010)$. Similarly, dyspareunia is also a significantly more common complaint in the severe PFMP group than in other groups $(p=.031)$. These results are consistent with the results of a previous study. ${ }^{7}$

One previous study has revealed the high prevalence $(78 \%-87 \%)$ of concomitant myofascial pain, frequently extending beyond the anatomical boundaries of the pelvis in women with IC/BPS. Almost a quarter of women with chronic pelvic pain (CPP) have musculoskeletal abnormalities. ${ }^{22-25}$ Interestingly, the mean PFM trigger point pain scores in healthy control women were lower than that in CPP and IC/BPS subjects. ${ }^{24}$ It is likely that patients with CPP and IC/BPS might have more discomfort in the PFMs. Although the self-report IC/BPS symptom scores (ICSI, ICPI) are indeed relatively higher in the moderate to severe PFMP group $(p=.012)$, there is no difference in the MBC $(p=.505)$ and glomerulation grade $(p=.875)$ and changes of uroflowmetry parameters (changes of $p$-value, Qmax: .176; voiding volume: .311; PVR: .770) among different PFMP groups. The self-report IC/BPS symptom and problem index (OSS) might be more related to the psychological condition rather than the bladder conditions, causing an increase in the patient's anxiety status score (BAI) with PFMP. ${ }^{6}$

Likewise, the GRA score also has a significant association with different PFMP severities. This result has repeatedly demonstrated that self-report treatment outcomes are closely related to PFM conditions, such as in the ICSI $(p=.029), I C P I(p=.007)$, and even GRA ( $p=.015)$. Compared with another study reporting on CPP in women, PFMP was significantly higher than that in healthy women ( $58.3 \%$ vs. $4.2 \%, P<.001)$, and women with CPP had higher BAI score (22 vs. $13, \mathrm{P}=.02)$, higher dyspareunia rate $(63.5 \%$ vs. $28.9 \%$, $\mathrm{P}<.004)$, and higher constipation rate $(46.0 \%$ vs. $26.7 \%, \mathrm{P}=.05)$ than those without PFMP. ${ }^{10}$

As mentioned above, the HIC subtype is a distinct inflammatory disease with proven bladder etiology and characterized by epithelial denudation and enhanced immune responses. ${ }^{25}$ Meanwhile, the NHIC subtype is potentially associated with urothelial malfunction and neurophysiological dysfunction and frequently presents with somatic and/or psychological symptoms, which might result from psychosomatic disorders due to CNS sensitization. Furthermore, if CNS inflammation is the cause of bladder disorders in IC/BPS, the inflammatory responses in the CNS involve the participation of different cellular immune system and resident cells of the CNS, adhesion molecules, cytokines, and chemokines among other 
protein components. If this process is not well controlled or is prolonged, the bladder will ultimately lose its regenerative and repair function and can be the cause of urothelial damage and bladder dysfunction. ${ }^{26}$

In this study, $86.2 \%$ of patients with IC/BPS have PFMP, and patients with a higher pelvic pain severity also have more CNS sensitization comorbidities. However, the severity of pelvic pain is not significantly related to bladder conditions, such as $\mathrm{MBC}$, glomerulation grade, and even urodynamic parameters, but is significantly related to self-reported bladder symptom index. Moreover, the multiple linear regression analyses also show that PFMP severity is significantly correlated with patient's self-reported treatment outcome. The results of this study reveal that the PFMP could influence the treatment outcome of women with IC/BPS.

\section{Conclusion}

PFMP might affect the subjective results of IC/BPS treatment but not bladder conditions. Therefore, in the future treatment of patients with IC/BPS, digital vaginal examinations of PFMs should be performed in every patient with IC/BPS and focused more on PFM-related conditions, and necessary PFM treatments, such as the vaginal PFM message, should be scheduled.

\section{Declarations}

\section{Conflict of interest}

The authors declare no conflicts of interest.

\section{Data Availability}

To protect patient privacy and comply with relevant regulations, identified data are unavailable. Requests from qualified researchers with appropriate ethics board approvals and relevant data use agreements for de-identified data will be available.

\section{Acknowledgements}

This study was supported by TCGH IRB: 105-25-B from the Hualien Tzu Chi Hospital, Buddhist Tzu Chi Medical Foundation.

\section{Author contributions}

HCK and WRY conceived the study, made critical comments, and provided supervision. HCK was the principal investigator of this study and designed the study. WRY designed the study workflow and acquired, analyzed, and interoperated the data, in addition to drafting the article and making critical revisions. All authors reviewed the manuscript. 


\section{References}

1 Lukban, J. C. et al. Interstitial cystitis and pelvic floor dysfunction: a comprehensive review. Pain Medicine 2, 60-71 (2001).

2 Borrego-Jimenez, P.-S. et al. Improvement in Quality of Life with Pelvic Floor Muscle Training and Biofeedback in Patients with Painful Bladder Syndrome/Interstitial Cystitis. Journal of clinical medicine 10, 862 (2021).

3 Grinberg, K., Sela, Y., Nissanholtz-Gannot, R. J. I. J. o. E. R. \& Health, P. New Insights about Chronic Pelvic Pain Syndrome (CPPS). 17, 3005 (2020).

$4 \quad$ Kraemer, S., Watson, B. \& Peters, K. M. The Evaluation and Management of Interstitial Cystitis/Bladder Pain Syndrome. UROLOGY (2019).

5 Jiang, Y.-H. \& Kuo, H.-C. Urothelial Dysfunction and Increased Suburothelial Inflammation of Urinary Bladder Are Involved in Patients with Upper Urinary Tract Urolithiasis-Clinical and Immunohistochemistry Study. PLoS One 9, e110754 (2014).

6 Yu, W. R., Peng, T. C., Yeh, H. L. \& Kuo, H. C. Anxiety severity does not influence treatment outcomes in patients with interstitial cystitis/bladder pain syndrome. Neurourology and urodynamics 38, 1602-1610 (2019).

7 van Reijn-Baggen, D. A. et al. Pelvic Floor Physical Therapy for Pelvic Floor Hypertonicity: A Systematic Review of Treatment Efficacy. Sexual Medicine Reviews (2021).

8 Bartley, J. et al. Transvaginal trigger point injections improve pain scores in women with pelvic floor hypertonicity and pelvic pain conditions. Female pelvic medicine \& reconstructive surgery $25,392-$ 396 (2019).

9 Meister, M. R., Sutcliffe, S., Badu, A., Ghetti, C. \& Lowder, J. L. Pelvic floor myofascial pain severity and pelvic floor disorder symptom bother: is there a correlation? American journal of obstetrics and gynecology 221, 235. e231-235. e215 (2019).

10 de Souza Montenegro, M. L. L. et al. Importance of pelvic muscle tenderness evaluation in women with chronic pelvic pain. 11, 224-228 (2010).

11 Gyang, A., Hartman, M. \& Lamvu, G. Musculoskeletal causes of chronic pelvic pain: what a gynecologist should know. Obstetrics \& Gynecology 121, 645-650 (2013).

12 Howard, F. M. Pelvic pain: diagnosis and management. (Lippincott Williams \& Wilkins, 2000).

13 Gerwin, R. D. Myofascial pain syndromes from trigger points. Current review of pain 3, 153-159 (1999). 
14 Butrick, C. W. Interstitial cystitis and chronic pelvic pain: new insights in neuropathology, diagnosis, and treatment. Clinical obstetrics and gynecology 46, 811-823 (2003).

15 Hanno, P. M. \& Sant, G. R. Clinical highlights of the National Institute of Diabetes and Digestive and Kidney Diseases/Interstitial Cystitis Association scientific conference on interstitial cystitis. Urology 57, 2-6 (2001).

16 Meister, M. R. et al. Development of a standardized, reproducible screening examination for assessment of pelvic floor myofascial pain. American journal of obstetrics and gynecology 220, 255. e251-255. e259 (2019).

17 Beck, A., Epstein, N., Brown, G. \& Steer, R. An inventory for measuring clinical anxiety: The Beck Anxiety Inventory. J Consult Clin Psychol 56, 893-897 (1988).

18 Hanno, P. M., Nordling, J., Staskin, D. R., Wein, A. J. \& Wyndaele, J. J. Bladder Pain Syndrome-An Evolution. (Springer, 2018).

19 Jhang, J.-F. et al. Epstein-Barr virus as a potential etiology of persistent bladder inflammation in human interstitial cystitis/bladder pain syndrome. The Journal of urology 200, 590-596 (2018).

20 Akiyama, Y. Update on the pathophysiology of interstitial cystitis/bladder pain syndrome. Current Bladder Dysfunction Reports 15, 1-8 (2020).

21 Irwin, M. R. \& Cole, S. W. Reciprocal regulation of the neural and innate immune systems. Nature Reviews Immunology 11, 625-632 (2011).

22 Chelimsky, G. et al. Co-morbidities of interstitial cystitis. Frontiers in neuroscience 6, 114 (2012).

23 Wang, Y. \& Kasper, L. H. The role of microbiome in central nervous system disorders. Brain, behavior, and immunity 38, 1-12 (2014).

24 Sanses, T. V. et al. The pelvis and beyond: musculoskeletal tender points in women with chronic pelvic pain. 32, 659 (2016).

25 Akiyama, Y., Luo, Y., Hanno, P. M., Maeda, D. \& Homma, Y. Interstitial cystitis/bladder pain syndrome: The evolving landscape, animal models and future perspectives. International Journal of Urology 27, 491-503 (2020).

26 Chavarria, A. \& Alcocer-Varela, J. Is damage in central nervous system due to inflammation? Autoimmunity reviews 3, 251-260 (2004).

\section{Tables}


Table 1. Correlation between subjective measured parameters of IC/BPS among different PFM pain severities from vaginal digital examination $(n=65)$

\begin{tabular}{|c|c|c|c|c|c|}
\hline \multicolumn{2}{|c|}{ PFM pain severity (VAS) } & Mild & Moderate & \multirow{3}{*}{$\begin{array}{l}\text { Severe pain } \\
\text { VAS } 7-10 \\
(n=42)\end{array}$} & \multirow{3}{*}{$\begin{array}{l}\mathrm{P} \text { - } \\
\text { value }\end{array}$} \\
\hline & & $\begin{array}{l}\text { VAS 0- } \\
3\end{array}$ & $\begin{array}{l}\text { VAS 4-6 } \\
(n=11)\end{array}$ & & \\
\hline & & \multicolumn{2}{|l|}{$(n=12)$} & & \\
\hline \multicolumn{2}{|l|}{ Age } & $\begin{array}{l}55.2 \pm \\
13.9\end{array}$ & $52.4 \pm 8.7$ & $58.8 \pm 10.9$ & .199 \\
\hline \multirow{4}{*}{$\begin{array}{l}\text { Sexual } \\
\text { activity }\end{array}$} & Continue to have sex & $7(58.4 \%)$ & $5(45.5 \%)$ & 15(35.7\%) & \multirow[t]{4}{*}{.734} \\
\hline & $\begin{array}{l}\text { No sexual intercourse within a } \\
\text { year }\end{array}$ & $1(8.3 \%)$ & $0(.0 \%)$ & $2(4.8 \%)$ & \\
\hline & $\begin{array}{l}\text { No sexual intercourse within } 3 \\
\text { years }\end{array}$ & $1(8.3 \%)$ & $1(9.0 \%)$ & $2(4.8 \%)$ & \\
\hline & $\begin{array}{l}\text { No sexual intercourse more } \\
\text { than } 5 \text { years }\end{array}$ & $3(25.0 \%)$ & $5(45.5 \%)$ & $23(54.7 \%)$ & \\
\hline \multicolumn{2}{|c|}{ Dyspareunia } & $2(16.7 \%)$ & $5(45.5 \%)$ & $25(59.5 \%)$ & $.031^{\star}$ \\
\hline \multicolumn{2}{|c|}{ Duration of IC/BPS } & $\begin{array}{l}10.4 \pm \\
10.1\end{array}$ & $9.9 \pm 6.5$ & $14.2 \pm 11.3$ & .340 \\
\hline \multicolumn{2}{|c|}{ Transvaginal surgery experience } & $5(41.7 \%)$ & $3(27.3 \%)$ & $21(50.0 \%)$ & .392 \\
\hline \multicolumn{2}{|c|}{ Comorbidities } & $.7 \pm .7$ & $2.6 \pm 1.7$ & $2.2 \pm 1.8$ & $.010^{\star}$ \\
\hline \multicolumn{2}{|c|}{ Type of treatment } & $3.7 \pm 1.3$ & $3.2 \pm .9$ & $3.2 \pm 1.4$ & .513 \\
\hline \multicolumn{2}{|c|}{ Number of PFMP sites } & $.8 \pm 1.4$ & $3.6 \pm 1.5$ & $6.8 \pm 4.4$ & $<.001 *$ \\
\hline \multicolumn{2}{|l|}{ BAI } & $\begin{array}{l}15.3 \pm \\
8.3\end{array}$ & $19.5 \pm 11.5$ & $23.7 \pm 12.6$ & .082 \\
\hline \multicolumn{2}{|l|}{ ICSI } & $\begin{array}{l}10.1 \pm \\
4.9\end{array}$ & $14.1 \pm 5.0$ & $13.8 \pm 4.0$ & $.029 *$ \\
\hline \multicolumn{2}{|l|}{ ICPI } & $9.8 \pm 3.8$ & $13.0 \pm 4.4$ & $13.5 \pm 3.0$ & $.007 *$ \\
\hline \multicolumn{2}{|l|}{ OSS } & $\begin{array}{l}19.9 \pm \\
8.4\end{array}$ & $27.1 \pm 9.2$ & $27.3 \pm 6.6$ & $.012^{\star}$ \\
\hline \multicolumn{2}{|c|}{$\begin{array}{l}\text { NRS (self-reported lower abdomen pain } \\
\text { severity) }\end{array}$} & $4.7 \pm 3.2$ & $6.7 \pm 2.6$ & $6.4 \pm 2.3$ & .093 \\
\hline \multicolumn{2}{|l|}{ GRA } & $2.2 \pm .9$ & $1.6 \pm .7$ & $.9 \pm 1.5$ & $.015^{\star}$ \\
\hline
\end{tabular}

IC/BPS, interstitial cystitis/bladder pain syndrome; PFM, pelvic floor muscle; VAS, visual analog scale; BAI, Beck's anxiety inventory; ICSI, interstitial cystitis symptom index; ICPI, interstitial cystitis problem 
index; OSS, O'Leary-Sant score; NRS, numerical rating scale; GRA, global response assessment; ${ }^{\star} \mathrm{p}<.05$

Table 2. Correlation between objective measured parameters of IC/BPS among different PFM pain severity from vaginal digital examination $(n=65)$ 


\begin{tabular}{|c|c|c|c|c|c|c|}
\hline \multirow{2}{*}{\multicolumn{3}{|c|}{ PFM pain severity (VAS) }} & Mild pain & Moderate & Severe & P-value \\
\hline & & & $(n=12)$ & $\begin{array}{l}\text { VAS 4-6 } \\
(n=11)\end{array}$ & $\begin{array}{l}\text { VAS 7- } \\
10 \\
(n=42)\end{array}$ & \\
\hline \multicolumn{3}{|l|}{ MBC } & $\begin{array}{l}750.0 \pm \\
179.6\end{array}$ & $\begin{array}{l}804.6 \pm \\
179.5\end{array}$ & $\begin{array}{l}816.3 \pm \\
166.9\end{array}$ & .505 \\
\hline \multicolumn{3}{|c|}{ Glomerulation grade } & $1.5 \pm 1.2$ & $1.3 \pm .9$ & $1.3 \pm 1.2$ & .875 \\
\hline \multirow{3}{*}{\multicolumn{2}{|c|}{ Bladder CT finding }} & Smooth & $10(83.4 \%)$ & $5(50.0 \%)$ & $27(65.9 \%)$ & \multirow[t]{3}{*}{.474} \\
\hline & & Focal & $1(8.3 \%)$ & $4(40.0 \%)$ & $9(22.0 \%)$ & \\
\hline & & diffuse & $1(8.3 \%)$ & $1(10.0 \%)$ & $5(12.1 \%)$ & \\
\hline \multirow[t]{4}{*}{$\begin{array}{l}\text { VUDS } \\
\text { parameter }\end{array}$} & \multicolumn{2}{|l|}{ FSF } & $\begin{array}{l}125.9 \pm \\
38.0\end{array}$ & $\begin{array}{l}122.5 \pm \\
46.2\end{array}$ & $\begin{array}{l}116.8 \pm \\
46.4\end{array}$ & .800 \\
\hline & \multicolumn{2}{|l|}{ FS } & $\begin{array}{l}217.1 \pm \\
81.7\end{array}$ & $\begin{array}{l}194.6 \pm \\
62.9\end{array}$ & $\begin{array}{l}197.3 \pm \\
74.5\end{array}$ & .688 \\
\hline & \multicolumn{2}{|l|}{ CBC } & $\begin{array}{l}254.8 \pm \\
91.5\end{array}$ & $\begin{array}{l}243.6 \pm \\
66.2\end{array}$ & $\begin{array}{l}259.8 \pm \\
126.9\end{array}$ & .914 \\
\hline & \multicolumn{2}{|l|}{ Pdet } & $15.5 \pm 7.3$ & $\begin{array}{l}19.3 \pm \\
6.4\end{array}$ & $\begin{array}{l}19.3 \pm \\
12.6\end{array}$ & .569 \\
\hline \multirow[t]{4}{*}{$\mathrm{KCL}$ test } & \multicolumn{2}{|l|}{ Negative } & $2(16.7 \%)$ & $0(.0 \%)$ & $2(4.8 \%)$ & \multirow[t]{4}{*}{.065} \\
\hline & \multicolumn{2}{|c|}{ Positive with pain } & $7(58.3 \%)$ & $7(63.6 \%)$ & $36(85.6 \%)$ & \\
\hline & \multicolumn{2}{|c|}{ Positive with urge } & $3(25.0 \%)$ & $2(18.2 \%)$ & $2(4.8 \%)$ & \\
\hline & \multicolumn{2}{|c|}{$\begin{array}{l}\text { Positive with pain and } \\
\text { urgency }\end{array}$} & $0(.0 \%)$ & $2(18.2 \%)$ & $2(4.8 \%)$ & \\
\hline \multirow[t]{5}{*}{ Uroflowmetry } & \multirow[t]{3}{*}{ Qmax } & Baseline & $12.3 \pm 5.5$ & $\begin{array}{l}10.9 \pm \\
5.9\end{array}$ & $11.1 \pm 5.5$ & .771 \\
\hline & & $\begin{array}{l}\text { After treatment } \\
1 \mathrm{M}\end{array}$ & $13.8 \pm 8.3$ & $\begin{array}{l}16.7 \pm \\
6.4\end{array}$ & $\begin{array}{l}19.0 \pm \\
11.8\end{array}$ & .318 \\
\hline & & $\Delta$ & $1.5 \pm 8.7$ & $5.6 \pm 6.4$ & $7.9 \pm 11.5$ & .176 \\
\hline & \multirow[t]{2}{*}{$\begin{array}{l}\text { Voiding- } \\
\text { volume }\end{array}$} & Baseline & $\begin{array}{l}233.4 \pm \\
111.0\end{array}$ & $\begin{array}{l}214.2 \pm \\
85.0\end{array}$ & $\begin{array}{l}221.4 \pm \\
118.2\end{array}$ & .915 \\
\hline & & $\begin{array}{l}\text { After treatment } \\
1 \mathrm{M}\end{array}$ & $\begin{array}{l}172.8 \pm \\
72.9\end{array}$ & $\begin{array}{l}237.4 \pm \\
171.7\end{array}$ & $\begin{array}{l}197.3 \pm \\
104.6\end{array}$ & .392 \\
\hline
\end{tabular}




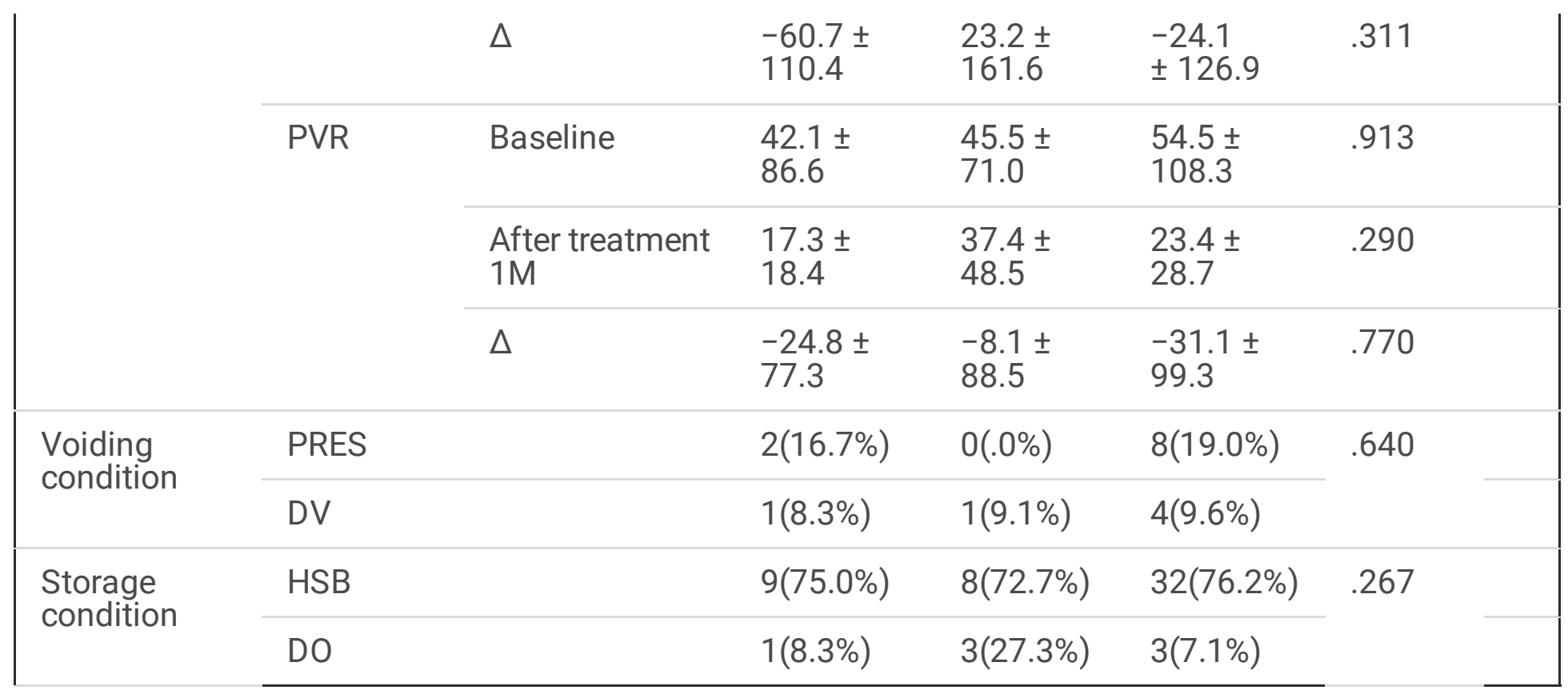

IC/BPS, interstitial cystitis/bladder pain syndrome; PFM, pelvic floor muscle; VAS, visual analog score; MBC, maximal bladder capacity; CT, computed tomography; VUDS, video urodynamic study; FSF, first sensation of filling; FS, full sensation; $C B C$, cytometric bladder capacity; Pdet, detrusor pressure; Qmax, maximum flow rate; PVR. post-void residual urine; PRES, poor relaxation of external urethral sphincter; DV, dysfunctional voiding; HSB, hypersensitivity bladder; DO, detrusor overactivity; ${ }^{\star} \mathrm{p}<.05$

Table 3. Multiple linear regression analyses on factors associated with PFM pain severity (VAS) $(n=65)$

\begin{tabular}{|lllll|}
\hline Constant & Coefficient $(\boldsymbol{\beta})$ & $\mathbf{9 5 \%} \mathbf{C l}$ & & P-value \\
\hline Age & -.059 & -.015 & .133 & .116 \\
\hline OSS & .096 & -.018 & .210 & .098 \\
BAl & -.001 & -.082 & .080 & .975 \\
Glomerulation grade & -.099 & -1.054 & .856 & .836 \\
GRA & -1.645 & -2.293 & -.996 & $<.001^{*}$ \\
MBC & -.001 & -.007 & .004 & .597 \\
\hline
\end{tabular}

OSS, O'Leary-Sant score; BAI, Beck's anxiety inventory; GRA, global response assessment; MBC, maximal bladder capacity; ${ }^{*} \mathrm{p}<.05$

\section{Figures}




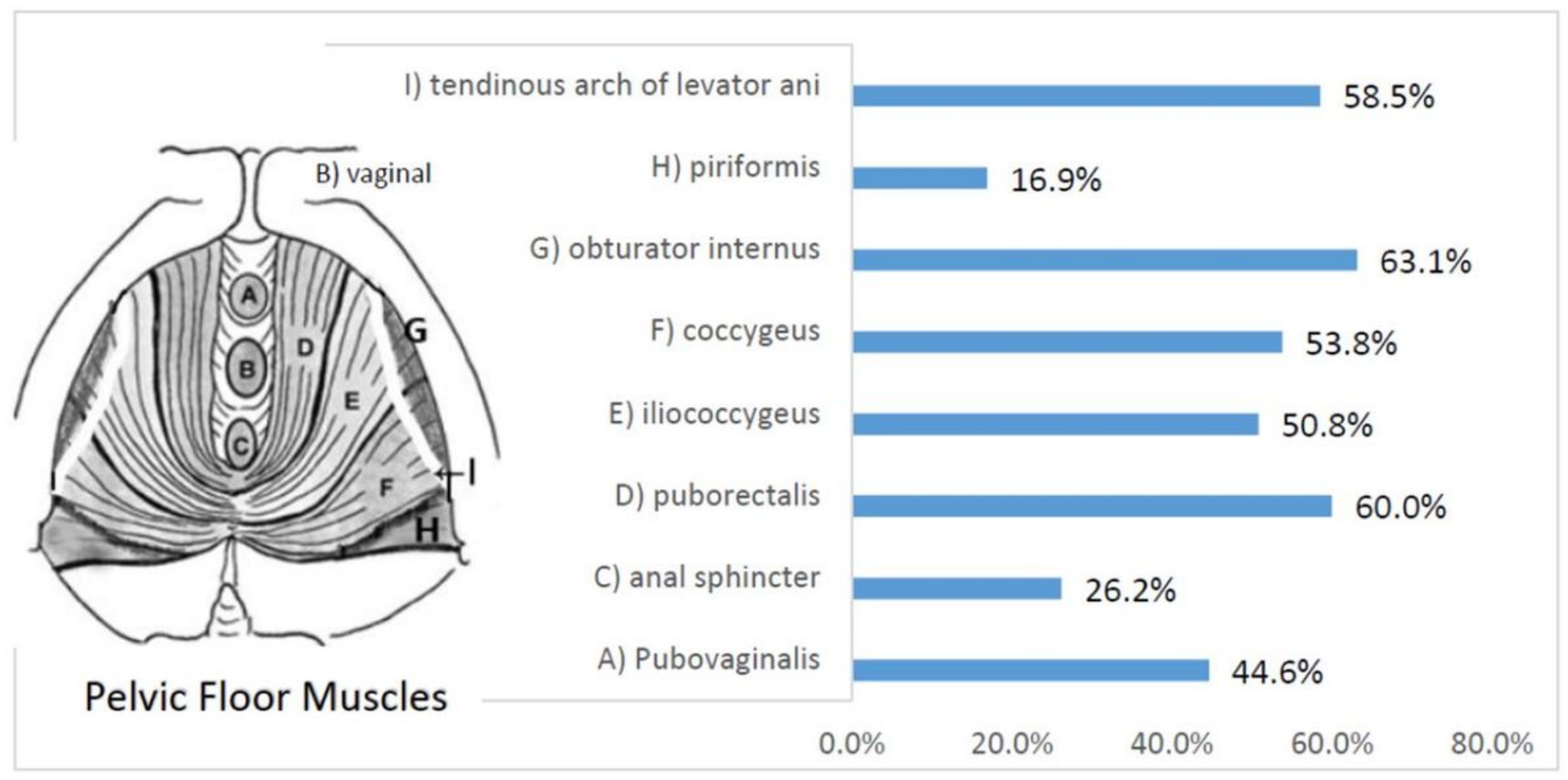

Figure 1

Pelvic floor muscle pain statistical chart $(n=65)$ 